\title{
Characterization of the Imitation Switch subfamily of ATP-dependent chromatin-remodeling factors in Saccharomyces cerevisiae
}

\author{
Toshio Tsukiyama, ${ }^{1,3,4}$ Jeffrey Palmer, ${ }^{1}$ Carolyn C. Landel, ${ }^{3}{ }^{\text {Joseph Shiloach, }}{ }^{2}$ and Carl Wu ${ }^{1}$ \\ ${ }^{1}$ Laboratory of Molecular Cell Biology, National Cancer Institute, and ${ }^{2}$ Biotechnology Unit, National Institute of Diabetes \\ and Digestive Kidney Diseases, National Institutes of Health, Bethesda, Maryland 20892 USA; ${ }^{3}$ Division of Basic Sciences, \\ Fred Hutchinson Cancer Research Center, Seattle, Washington 98109-1024 USA
}

We have identified and characterized two Imitation Switch genes in Saccharomyces cerevisiae, ISW1 and ISW2, which are highly related to Drosophila ISWI, encoding the putative ATPase subunit of three ATP-dependent chromatin remodeling factors. Purification of ISW1p reveals a four-subunit complex with nucleosome-stimulated ATPase activity, as well as ATP-dependent nucleosome disruption and spacing activities. Purification of ISW2p reveals a two-subunit complex also with nucleosome-stimulated ATPase and ATP-dependent nucleosome spacing activities but no detectable nucleosome disruption activity. Null mutations of ISW1, ISW2, and CHD1 genes cause synthetic lethality in various stress conditions in yeast cells, revealing the first in vivo functions of the ISWI subfamily of chromatin-remodeling complexes and demonstrating their genetic interactions. A single point mutation within the ATPase domain of both ISW1p and ISW2p inactivated all ATP-dependent biochemical activities of the complexes, as well as the ability of the genes to rescue the mutant phenotypes. This demonstrates that the ATP-dependent chromatin-remodeling activities are essential for the in vivo functions of both ISW1 and ISW2 complexes.

[Key Words: S. cerevisiae; ISW1; ISW2; chromatin-remodeling factors]

Received December 30, 1998; revised version accepted January 28, 1999.

In eukaryotic cells the genome is packaged into chromatin, a large nucleoprotein complex (van Holde 1988). The primary unit of chromatin is the nucleosome, which has $\sim 146$ bp of DNA wrapped around two copies each of core histone proteins $\mathrm{H} 2 \mathrm{~A}, \mathrm{H} 2 \mathrm{~B}, \mathrm{H} 3$, and $\mathrm{H} 4$ (Luger et al. 1997). Arrays of nucleosomes form further higher-order chromatin structures. Chromatin allows compact storage of DNA within the nucleus, but its highly condensed nature inhibits the interaction of proteins with DNA. Because protein-DNA interaction is an essential step in a wide variety of biological processes, it has been postulated that mechanisms that facilitate specific proteinDNA interactions occur on chromatin in vivo. This, in turn, implies that the mechanisms that regulate chromatin structure may play critical roles in the regulation of those processes. Recent studies have revealed that the factors affecting assembly and remodeling of chromatin structure also affect the regulation of transcription (Kornberg and Lorch 1995; Felsenfeld 1996; Kingston et al. 1996; Krude and Elgin 1996; Steger and Workman

${ }^{4}$ Corresponding author.

E-MAIL ttsukiya@fhcrc.org; FAX (206) 667-6497.
1996; Svaren and Horz 1996; Tsukiyama and Wu 1997; Wolffe et al. 1997; Armstrong and Emerson 1998; Kadonaga 1998), recombination (Wu and Lichten 1994; Fan and Petes 1996; Stanhope-Baker et al. 1996; Mizuno et al. 1997), and DNA repair (Gaillard et al. 1996; Kaufman et al. 1997; Tsukamoto et al. 1997), all of which require protein-DNA interactions. Elucidation of the mechanisms of assembly and remodeling of chromatin structure is therefore a necessary prerequisite in understanding how these processes are regulated.

Biochemical and genetic experiments in the past decade have identified a number of mechanisms by which chromatin structure can be altered to allow specific protein-DNA interactions to take place. Among those, we have been interested in ATP-dependent chromatin-remodeling factors (Peterson 1996; Pazin and Kadonaga 1997; Armstrong and Emerson 1998; Cairns 1998). ATPdependent chromatin remodeling in vitro was first discovered in transcription factor-mediated disruption of nucleosome arrays (Tsukiyama et al. 1994). Previously identified SWI/SNF complexes were then shown to be ATP-dependent chromatin-remodeling factors /Cote et al. 1994; Imbalzano et al. 1994; Kwon et al. 1994; Wang et al. 1996a,b). Subsequent studies identified NURF 
(nucleosome remodeling factor) (Tsukiyama and $\mathrm{Wu}$ 1995), RSC (remodeling structure of chromatin) (Cairns et al. 1996), CHRAC (chromatin accessibility complex) (Varga-Weisz et al. 1997), ACF (ATTP-utilizing chromatin assembly and remodeling factor) (Ito et al. 1997), Mi-2 complex (Wade et al. 1998; Zhang et al. 1998), and RSF (remodeling and spacing factor) (LeRoy et al. 1998) as ĀTP-dependent chromatin-remodeling complexes. Interestingly, all of the ATP-dependent chromatin-remodeling factors identified thus far have the putative ATPase subunit that belongs to the SWI2/SNF2 superfamily (Eisen et al. 1995). NURF (Tsukiyama et al. 1995), CHRAC (Varga-Weisz et al. 1997), and ACF (Ito et al. 1997) share Drosophila ISWI (Imitation Switch) protein (Elfring et al. 1994) as a putative ATPase subunit. The Drosophila ISWI gene was originally cloned based on its homology within the ATPase domain to the Brahma gene (Tamkun et al. 1992), the SWI2/SNF2 homolog in flies. Phylogenetic studies revealed that the ISWI genes are highly conserved during evolution and form a distinct subfamily within the SWI2/SNF2 superfamily (Eisen et al. 1995). This suggests that the ISWI genes have indispensable functions in vivo.

NURF facilitates transcription factor-mediated disruption of physiologically spaced nucleosomal arrays. This leads to the establishment of accessible chromatin structure at the promoter regions of genes (Tsukiyama and Wu 1995) and facilitation of transcription from chromatin templates in vitro (Mizuguchi et al. 1997). CHRAC increases the access of restriction enzymes to chromatin DNA and facilitates regular spacing of nucleosomes (Varga-Weisz et al. 1997) as well as T antigen-dependent replication of SV40 DNA in vitro (Alexiadis et al. 1998). ACF facilitates both regular spacing of nucleosome arrays and transcription factor-mediated disruption of nucleosome arrays in the presence of histone chaperones (Ito et al. 1997). Taken together, currently available data suggest that those ATP-dependent chromatin-remodeling factors play critical roles in the regulation of chromatin structure. However, their in vivo functions and the mechanisms of their actions remain largely unknown. The functional specificity of distinct ATP-dependent chromatin-remodeling factors also remains as an intriguing and important question.

To address these issues, we chose to study chromatinremodeling factors in the budding yeast Saccharomyces cerevisiae, because powerful genetic and biochemical tools are available in this model system. In this report we identified two yeast ISWI genes, ISW 1 and ISW2, and purified and characterized the complexes containing their gene products. We found that ISW1p and ISW2p form distinct ATP-dependent chromatin-remodeling complexes with distinct biochemical activities. We also present the first evidence that the yeast ISWI genes are essential for cells to resist various stresses in vivo and that they show genetic interactions. Our biochemical and genetic experiments using mutant yeast ISWI genes indicate that the ATP-dependent chromatin-remodeling activities of the yeast ISWI complexes are essential for their in vivo functions.

\section{Results}

Two ISWI genes exist in S. cerevisiae

As a first step in isolating ATP-dependent chromatinremodeling factors in yeast, we sought to identify the yeast homolog of the Drosophila ISWI gene. A BLAST search using the entire amino-acid sequence of Drosophila ISWI against all translated open reading frames (ORFs) in the yeast genome identified several ORFs encoding proteins with significant homology (BLAST $P$-value $<10^{-50}$ ) to Drosophila ISWI. This suggested that these genes might have similar or partially redundant functions. Among those, two uncharacterized ORFs, YBR245C and YOR304W, encode proteins with significantly higher homologies to Drosophila ISWI than the other genes identified (Fig. 1A). The homology is not restricted to the highly conserved ATPase domain but extends across the entire molecule including the SANT (SWI3, ADA2, N-CoR, and TFIIIB B ${ }^{\prime \prime}$ ) domain, which is conserved in a wide variety of regulators of chromatin and gene expression (Aasland et al. 1996). We therefore concluded that YBR245C and YOR304W are the structural homologs of Drosophila ISWI in S. cerevisiae and named them ISW1 and ISW2, respectively. The homology of ISW1p and ISW2p to Drosophila ISWI is similar (BLAST $P$-value: $4 \times 10^{-200}$ and $1.9 \times 10^{-241}$, respectively).

Two yeast ISWI proteins form distinct complexes in vivo

Both ISW1p and ISW2p were purified from yeast cells to test if they form ATP-dependent chromatin-remodeling complexes. Both of the proteins were fused with the Flag epitope tag sequence (Asp-Tyr-Lys-Asp-Asp-Asp-AspLys) and expressed in yeast cells carrying a deletion of the corresponding ISWI gene on the chromosome, so that all ISWI protein in the cells harbors the epitope tag. The Flag epitope tag enabled us to purify both ISW1p and ISW2 $p$ to near homogeneity by three chromatographic steps (see Materials and Methods) (Fig. 1B). Purified ISW $1 p$ fraction has three proteins in addition to ISW $1 p$, p110, p105, and p74. These proteins copurify with ISW1p in several independent preparations and comigrate with the ATP-dependent biochemical activities of the ISW1p described below. We therefore concluded that the ISW1p is part of a four-subunit complex. Similarly, the ISW2 protein always copurifies with another protein, p140, to form a two-subunit complex. Thus far, no other complexes containing either ISW1p or ISW2p have been detected. We therefore believe that ISW1p and ISW2p form distinct complexes that do not share any common subunits.

Both of the yeast ISWI complexes have nucleosome-stimulated ATPase activities

ATP-dependent chromatin-remodeling complexes possess ATPase activities that are believed to be essential 
Figure 1. Yeast has two ISWI genes. (A) Schematic representation of the yeast genes homologous to Drosophila ISWI. Drosophila ISWI is presented at top. Domains with significant homologies are indicated by boxes with the same patterns. The ATPase domains and the carboxyterminal homologous domains of ISWI proteins are represented by solid and checkered boxes, respectively. The numbers below these domains indicate the length of the domains and homologies to the corresponding domain of Drosophila ISWI protein. Location of the SANT domain in ISWI proteins and the chromodomain and putative DNA-binding domain in CHD1p are indicated above the proteins. (B) ISW1 and ISW2 proteins form distinct complexes. The ISW1 and ISW2 complexes (Mono Q fractions) were separated through $10 \%$ SDS-PAGE and stained by silver. (Arrows) Subunits of the complexes. (O) Minor bands that are likely due to proteolytic degradation because the intensity of the bands increases during freeze and thaw. (Asterisks) Minor contaminants, which are present in some, but not in other, preparations (see Fig. 5A).
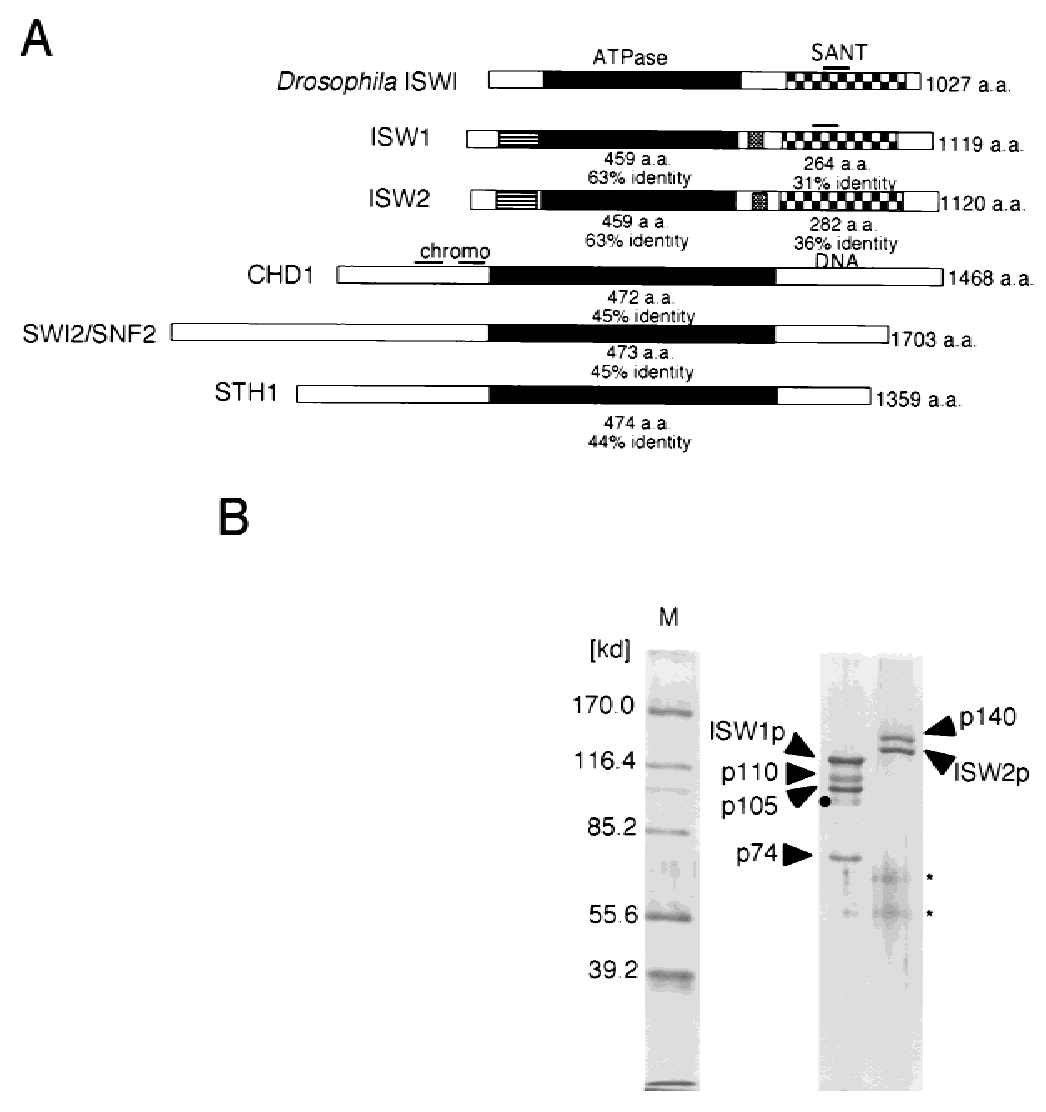

specifically from $\sim 9$ hydrolysis/complex per min in buffer to $\sim 130$ hydrolysis/complex per min in the presence of nucleosomes. We therefore concluded that both ISW1 and ISW2 complexes are able to recognize special structural features of nucleosomes.

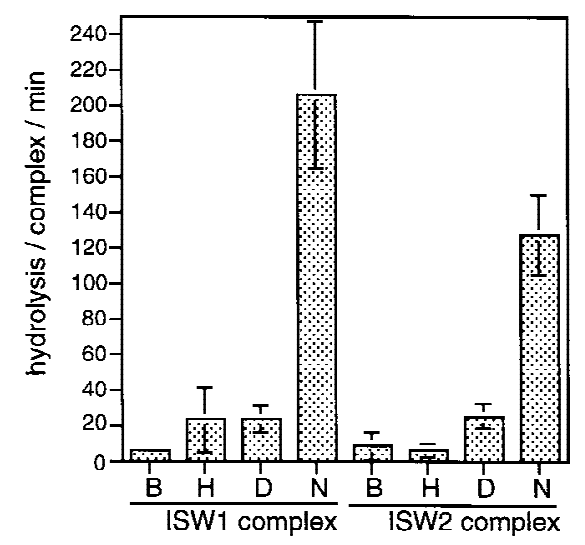

Figure 2. ISW1 and ISW2 complexes have nucleosome-stimulated ATPase activities. ATPase activities of ISW1 and ISW2 complexes were assayed in the presence of buffer (B), free core histones $(\mathrm{H})$, naked DNA (D), or nucleosomes (N). Hydrolysis/ complex/min denotes the molecules of ATP hydrolyzed by one molecule of the yeast ISWI complexes/min. Bars and vertical lines represent the average and the standard deviation, respectively, calculated from three independent experiments. 
The ISW1, but not the ISW2, complex disrupts nucleosome arrays in vitro

We next asked if these complexes are able to facilitate disruption of regularly spaced nucleosome arrays by sequence-specific transcription factors. NURF in flies facilitates disruption of nucleosome arrays by transcription factors to generate an active chromatin structure (Tsukiyama and Wu 1995; Mizuguchi et al. 1997). In this paper we call this activity 'nucleosome disruption activity' for convenience. We employed this system to directly compare the biochemical activities of ISW1 and ISW2 complexes with that of NURF. Regularly spaced nucleosome arrays were assembled onto a plasmid containing the hsp70 promoter in vitro using Drosophila embryo extract (Becker and Wu 1992; Becker et al. 1994). Plasmid chromatin was then treated with Sarkosyl to inactivate endogenous ATP-dependent factors and purified through a gel filtration column (Tsukiyama et al. 1994). After incubating the chromatin template with GAGA transcription factor and ISWI-containing complexes, the structure of the nucleosome array was analyzed by digestion with micrococcal nuclease (MNase) followed by Southern blotting as described previously (Tsukiyama and Wu 1996). When chromatin was purified without Sarkosyl pretreatment, endogenous ATPdependent factors in the chromatin assembly extract facilitated nucleosome remodeling at the promoter region as reported previously (Fig. 3A, top). Sarkosyl treatment abolished ATP- and GAGA factor-dependent nucleosome remodeling, which could be rescued by the addition of purified NURF as shown previously (Tsukiyama and $\mathrm{Wu}$ 1995). In this system appearance of a smear between nucleosome ladders upon partial MNase digestion and a decrease in mononucleosome signals, as well as appearance of subnucleosomal signals upon strong MNase digestion of chromatin, are hallmarks of nucleosome remodeling (Tsukiyama et al. 1994). A similar amount of ISW1 complex added to Sarkosyl-treated chromatin caused strong ATP-dependent nucleosome remodeling at the hsp70 promoter. This nucleosome remodeling took place specifically at the promoter region, as the nucleosome ladder remained unaffected within the coding region of the $h s p 70$ gene (Fig. 3A, bottom). Interestingly, the same amount of ISW2 complex did not cause any detectable nucleosome remodeling. This was the case even when a threefold excess of ISW2 complex was used (data not shown).

We then tested if either ISW1 or ISW2 complexes have the ability to increase the accessibility of chromatin at the promoter region of $h s p 70$. Chromatin prepared as above was incubated with GAGA factor and the yeast ISWI complexes and then with the restriction enzyme HaeII (Fig. 3B). HaeII has recognition sites just upstream $(-41)$ and downstream $(-22)$ of the TATA box. Compared to the naked DNA control, digestion of chromatin DNA by HaeII was strongly inhibited because of limited access of the enzyme to chromatin DNA. HaeII gained access to the promoter region when ATP, GAGA factor, and the endogenous ATP-dependent factors or purified NURF

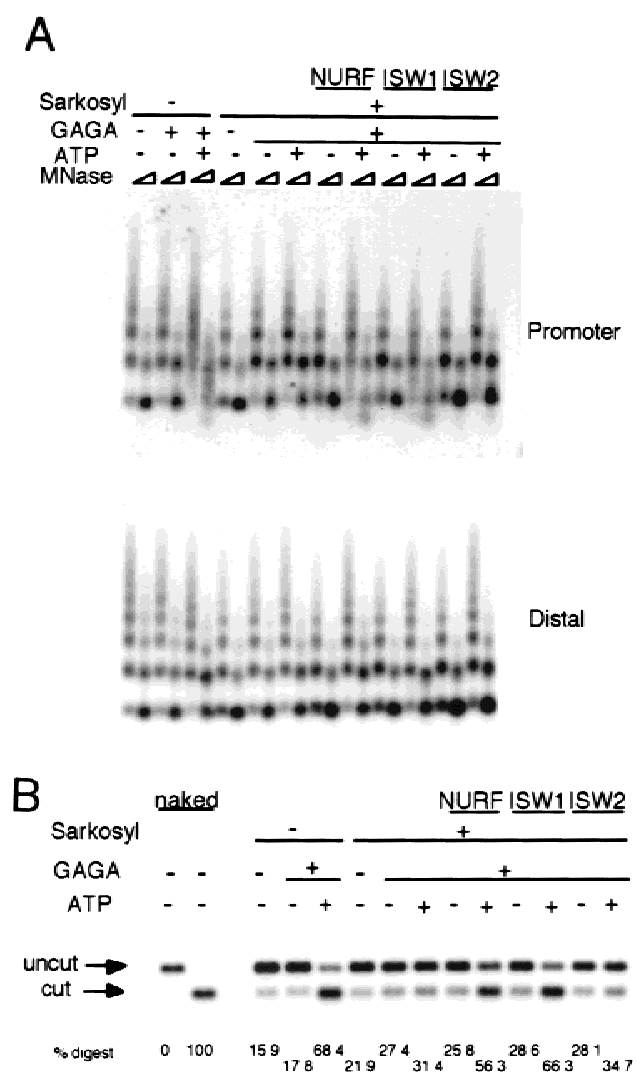

Figure 3. ISW1 complex has nucleosome disruption activity. (A) MNase digestion pattern of chromatin assembled on the plasmid containing the hsp70 gene. Purified NURF, ISW1, and ISW2 complexes were added to plasmid chromatin with or without GAGA factor and ATP. Partial and extended MNase digestions were performed for each sample. Oligonucleotide probes corresponding to the promoter and the distal regions of the hsp70 gene are as described (Tsukiyama et al. 1994). (B) Restriction enzyme accessibility assay at the promoter region of $h s p 70$ gene. Percentages of digested templates are indicated at bottom. Oligonucleotide probe used for hybridization is the same as the promoter probe in $A$.

were present. Similarly, the action of ISW1 complex and GAGA factor significantly increased the accessibility of HaeII at the TATA box region in an ATP-dependent manner. The increased accessibility of the restriction enzyme by ISW1 complex was restricted to the promoter region and was dependent on the presence of GAGA factor (data not shown). This activity, therefore, seems to be distinct from that of CHRAC complex (Varga-Weisz et al. 1997), which facilitates access of restriction enzymes on the entire plasmid chromatin, in the absence of DNA-binding factors. Consistent with the MNase digestion experiments above, ISW2 complex did not facilitate HaeII digestion. From these experiments, we concluded that ISW1 complex has an ATP-dependent nucleosome disruption activity. In contrast, ISW2 complex, despite its nucleosome-stimulated ATPase activity, does not exhibit the same activity in this system. 
Two yeast ISWI complexes have distinct nucleosome spacing activities

Two facts prompted us to test whether ISW1 and ISW2 complexes have activities that affect the spacing of nucleosomes. We frequently observed slight changes in the spacing of nucleosomes in the presence of yeast ISWI complexes during MNase ladder assays, as shown in Figure 3A. In addition, CHRAC (Varga-Weisz et al. 1997) and ACF (Ito et al. 1997) complexes, two Drosophila ISWI-containing complexes, have biochemical activities that change the spacing of nucleosomes. To assay for spacing activity in the yeast ISWI complexes, we employed an in vitro nucleosome assembly system using yeast nucleosome assembly protein, NAP-1 (Fujii-Nakata et al. 1992). NAP-1 acts as a histone chaperone by binding histones $\mathrm{H} 2 \mathrm{~A}$ and $\mathrm{H} 2 \mathrm{~B}$ and deposits core histones onto naked DNA at a physiological salt concentration in a randomly spaced fashion (Ito et al. 1996). MNase digestion of nucleosome arrays reconstituted by this method produced a smear over mono- and dinucleosome bands, showing that the spacing of nucleosomes is irregular (Fig. 4A). The intensity of nucleosome bands under this condition was somewhat variable, but the size of dinucleosome signal was always $\sim 300 \mathrm{bp}$. In contrast, MNase digestion of chromatin assembled in the presence of ISW1 complex and ATP produced discrete ladders, showing that nucleosomes are more regularly spaced. The average spacing between nucleosomes created by ISW1 complex in this system was $\sim 175 \mathrm{bp}$. The assembly of regularly spaced nucleosomes using ISW1 complex is dependent on the presence of ATP, core histones, and NAP-1. ISW2 complex also affected nucleosome spacing in an ATP-dependent manner. Interestingly, however, ISW2 and ISW1 complexes seem to have different activities. The nucleosome ladders created by ISW2 complex were reproducibly less discrete than those created by ISW1 complex. In addition, the spacing of nucleosomes created by ISW2 complex was $\sim 200 \mathrm{bp}$. We changed the amount of core histones, NAP-1, and the yeast ISWI complexes in nucleosome assembly reactions, but the spacing of nucleosomes was not affected by these parameters (data not shown). ISW2 complex also seems to facilitate nucleosome assembly as revealed by more prominent mononucleosome signals after strong MNase digestion, which was not the case in the reactions using ISW1 complex. In contrast to nucleosome disruption activity, Drosophila NURF did not exhibit significant nucleosome spacing activity (VargaWeisz et al. 1997; T. Tsukiyama, unpubl.), suggesting that yeast ISWI complexes may have biochemical properties distinct from that of NURF.

To determine the stoichiometry of yeast ISWI complexes to nucleosomes in nucleosome spacing reactions, increasing amounts of the complexes were used in nucleosome spacing assays (Fig. 4B). Although the effect of the yeast ISWI complexes on nucleosome spacing could be observed at lower levels, the spacing activity was saturated at 10 and 15 fmoles of ISW1 and ISW2 complex, respectively, in the standard reaction using 250

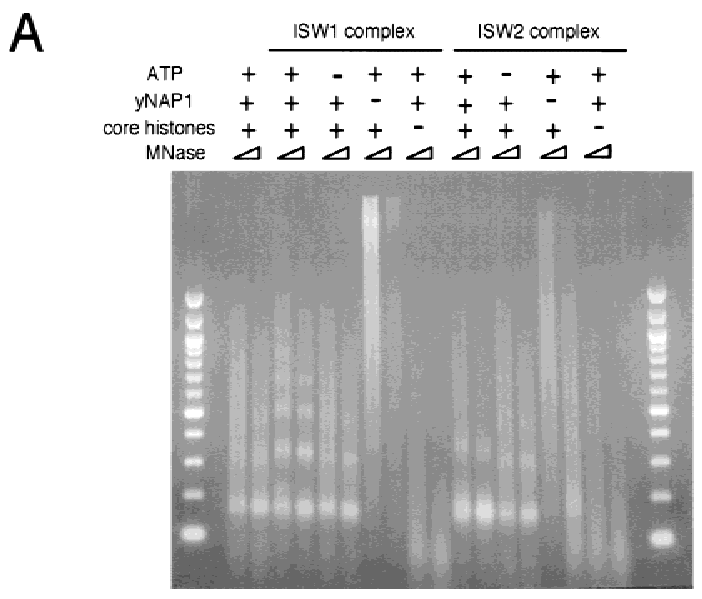

B

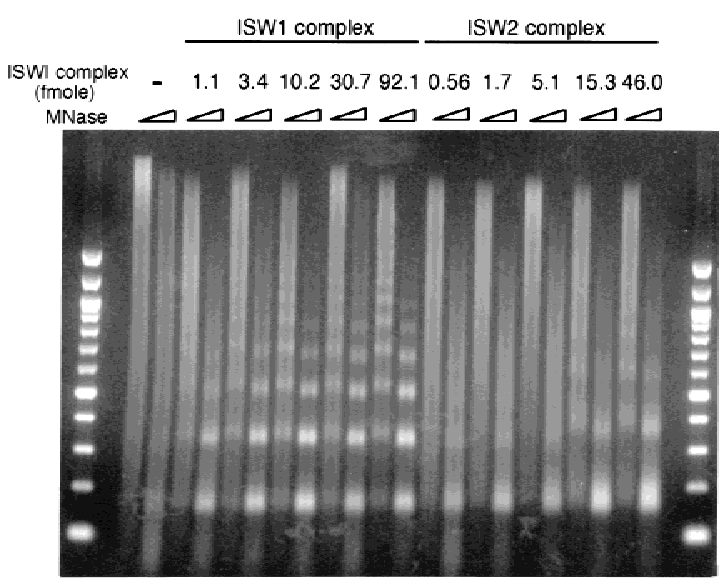

Figure 4. The yeast ISWI complexes have distinct nucleosome spacing activities. (A) Components required for the assembly of regularly spaced nucleosome arrays using the yeast ISWI complexes. Presence $(+)$ and absence $(-)$, respectively, of the components are indicated. Partial and extended MNase digestions were taken for each sample. (B) Titration of the yeast ISWI complexes in nucleosome spacing assay. Threefold serial dilution of the yeast ISWI complexes was used. All reactions contained NAP1, core histones, $\lambda$ DNA, and ATP at standard concentrations. Partial and extended MNase digestions were performed for each sample.

ng each of core histones and DNA. If we assume that the entire DNA template is saturated by regularly spaced nucleosomes, the ratio of the yeast ISWI complexes to nucleosomes under these conditions is approximately 1 molecule of ISW1 and ISW2 complex to 200 and 125 nucleosomes, respectively.

\section{Single point mutations inactivate the ATP-dependent activities of the ISWI complexes}

To create tools for studying the in vivo functions of the yeast ISWI complexes in yeast, we introduced a single amino acid substitution within the ATP binding site of both ISW1p and ISW2p (K227A for the ISW1p and K214A 
for the ISW2p). We chose these mutations because the analogous mutations in yeast (Laurent et al. 1993; Cote et al. 1994) and human (Khavari et al. 1993) SWI2/SNF2 proteins have been shown to inactivate their functions in vivo. In this paper we refer to the complexes containing mutant ISW1p and ISW2p as ISW1-K227A and ISW2K214A complexes, respectively. For unknown reasons, both ISW1-K227A and ISW2-K214A complexes were more susceptible to protease degradation in vivo than the wild-type complexes. Because of this, the p105 subunit in purified ISW1-K227A complex was consistently less abundant than other subunits (Fig. 5A). In contrast, the p110 and p74 bands were more prominent in the ISW1-K227A complex than in the wild-type complex. This may be due to comigration of degradation products of higher molecular subunits with p110 and p74. The ISW2-K214A complex has a subunit composition identical to that of the wild-type counterpart.

Biochemical activities of the ISW1-K227A and ISW2K214A complexes were tested to observe the effects of the point mutations. The ATPase activities of both complexes were at background level and were not stimulated by nucleosomes (Fig. 5B). This experiment demonstrates that both ISW1p and ISW2p are essential for the ATPase activities of the complexes. When these complexes were added to regularly spaced nucleosome arrays together with GAGA factor, they failed to demonstrate any effects on chromatin structure in either the MNase ladder assay (Fig. 5C) or the HaeII accessibility assay (Fig. 5D). The ISW1-K227A complex was also defective in spacing nucleosomes regularly as revealed by lack of regular nucleosome ladders in the nucleosome spacing assay in the presence of this complex (Fig. 5E). The ISW2-K214A complex was also defective in changing nucleosome spacing and facilitating nucleosome assembly as revealed by lack of shift in dinucleosome bands and lack of enhancement of mononucleosome signals in the presence of this complex. Importantly, the presence or absence of ATP did not affect chromatin structure in any of assays above when mutant complexes were used. From these data, we concluded that a single amino acid substitution introduced in either of the yeast ISWI proteins inactivated all ATP-dependent biochemical activities of the complexes tested. These data support our earlier conclusions that ATP is necessary for the yeast ISWI complexes to alter chromatin structure.

The loss of the ATP-dependent activities in the ISW1K227A complex is unlikely to be caused by a partial loss of the p105 subunit. We have experienced a similar loss of the p105 subunit in some preparations of the wildtype ISW1 complex. Whereas the subunit composition of these wild-type complexes was indistinguishable from
A

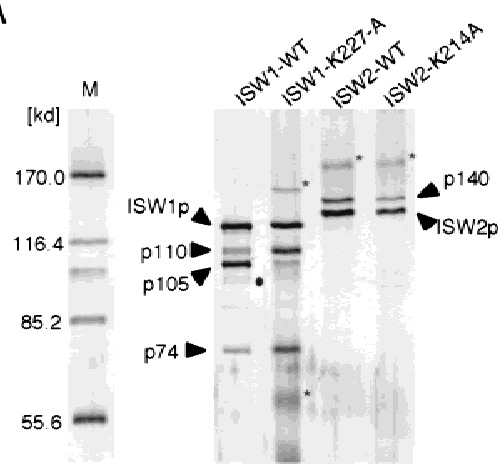

B

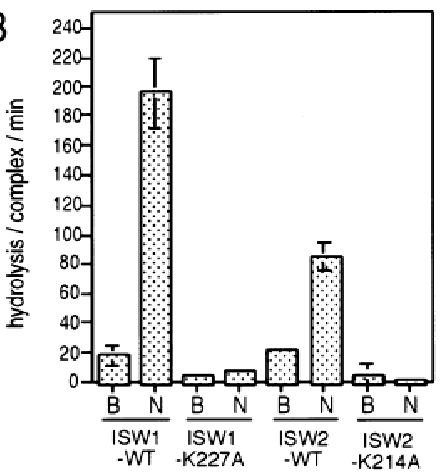

C

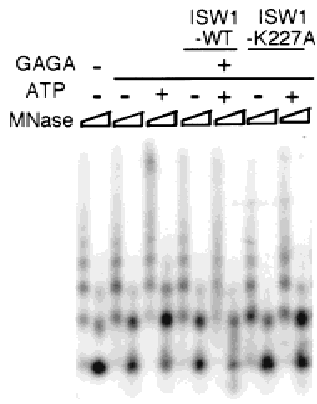

E

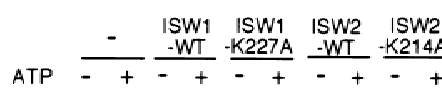

D

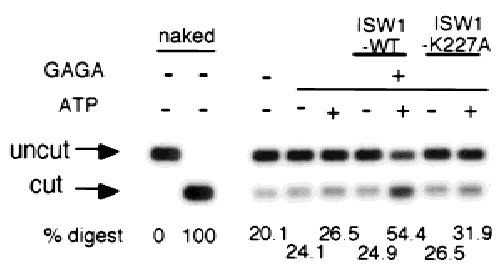

Figure 5. A single amino acid substitution within the ATPase domain of both yeast ISWI proteins inactivates ATP-dependent activities of the yeast ISWI complexes. (A) Subunit composition of the mutant yeast ISWI complexes. Purified wild-type and mutant yeast ISWI complexes were separated on an $8 \%$ SDS-polyacrylamide gel and stained by silver. (Arrows) The subunits of the complexes. Proteolytic products; $\left({ }^{\star}\right)$ contaminants. $(B)$ Mutant yeast ISWI complexes are defective in the ATPase activities. The ATPase assays were done in buffer (B) or in the presence of nucleosomes (N). Hydrolysis/complex per min denotes the molecules of ATP hydrolyzed by one molecule of the ISWI complex/ min. Bars and vertical lines represent the average and the standard deviation, respectively, calculated from three independent experiments. $(C-E)$ The mutant yeast ISWI complexes are defective in the MNase ladder assay, restriction enzyme accessibility assay, and nucleosome spacing assay, respectively. All assays were done in the standard conditions. For $C$ and $E$, partial and extended MNase digestions were performed for each sample. 
that of the ISW1-K227A complex used here, the complex was still active in all ATP-dependent assays tested (data not shown). We therefore believe that the loss of the ATP-dependent activities of the ISW1-K227A complex was caused by a point mutation within the ATP binding site per se rather than by a partial loss of the p105 subunit.

\section{Yeast ISWI genes show genetic interactions}

To begin to address the in vivo functions of the yeast ISWI complexes, we created yeast mutants that carry null alleles of the ISW1 and ISW2 genes by one-step gene replacement (Rothstein 1991) to observe phenotypes. Neither of the genes was essential for mitotic growth at normal conditions. Because there are multiple genes with significant homologies to the Drosophila ISWI gene in S. cerevisiae (Fig. 1A), we then tested if some of those genes show genetic interactions with either the ISW1 or ISW2 gene. We mutated the other nonessential genes that are homologous to the Drosophila ISWI gene, combined them with isw1 and/or isw2 mutations, and observed phenotypes. We tested mutants for sensitivities to various environmental stresses (heat shock survival, formamide, temperature and cold sensitivity, high salt, and caffeine); growth on galactose, raffinose, low phosphate and inositol-deficient medium; repair (sensitivity to UV, MMS, X-ray, and hydroxyurea); efficiency of mating and sporulation; stability of a CEN/ARS plasmid; and telomere position effect variegation (Gottschling et al. 1990). During a systematic survey of the phenotypes, we found that the triple mutant, isw1 isw2 chd1, shows a moderate growth defect at $37^{\circ} \mathrm{C}$ (Fig. 6A). This phenotype is synthetic, as none of the single null mutations of these genes caused any detectable growth defect at this temperature. This suggests that these three genes may have partially overlapping functions in vivo. At $38.5^{\circ} \mathrm{C}$, viability of the triple mutant, isw1 isw2 chd1, is $10^{-4}$ to $10^{-5}$ compared to wild-type cells. While the isw 1 mutant grew slightly slowly, single mutants do not exhibit strong growth defects at this temperature, showing a dramatic genetic interaction of ISW1, ISW2, and CHD1 genes. All combinations of double mutations caused significant growth defects at $38.5^{\circ} \mathrm{C}$. We observed a similar synthetic sensitivity of the isw1 isw2 chd1 mutants to formamide. However, the formamide-sensitivity phenotypes are different in that isw2 mutation has less effect on formamide sensitivity than on temperature sensitivity. We confirmed the 6AU-resistance phenotype of the chd1 mutant (Woodage et al. 1997) in our strain background. However, neither ISW1 nor ISW2 genes showed genetic interactions with the CHD1 gene regarding this phenotype (T. Tsukiyama, unpubl.).

To confirm the genetic interactions, we tested the rescue of the synthetic temperature-sensitive phenotype by transforming the isw1 isw2 chd1 triple mutant cells with a low copy number (CEN/ARS) vector expressing the ISWI and/or ISW2 genes from their own promoters. At $30^{\circ} \mathrm{C}$, cells transformed with an empty vector (pRS416) or yeast ISWI-expression vectors grew simi-
A

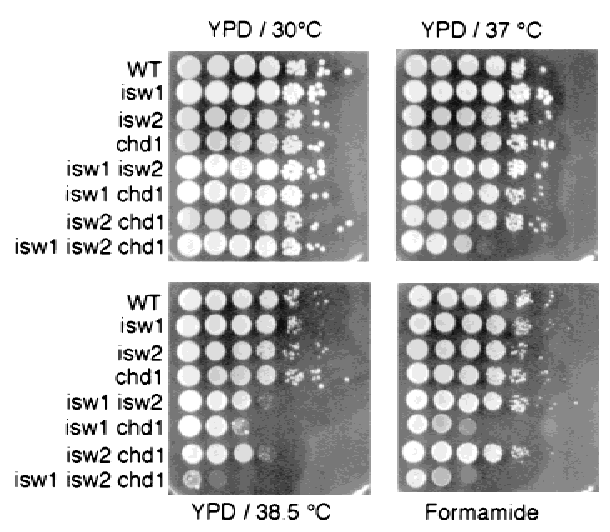

B

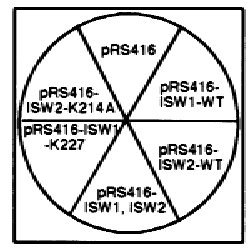

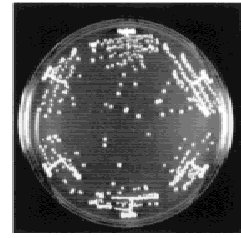

$30^{\circ} \mathrm{C}$

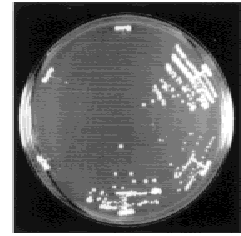

$37^{\circ} \mathrm{C}$
Figure 6. The yeast ISWI genes show genetic interactions. $|A|$ The synthetic stress-sensitive phenotypes of mutants. Wildtype and mutant cells were cultured to saturation, and tenfold dilutions were made for spotting. The medium and the temperatures used are indicated. Formamide plate, containing 3\% formamide in YPD, was incubated at $30^{\circ} \mathrm{C}$. Relevant genotypes of the mutants are indicated in on left. (B) Rescue of a synthetic temperature-sensitive phenotype of the isw1 isw2 chd1 mutant. The triple mutant was transformed with the plasmids indicated at left, streaked on synthetic medium lacking uracil, and incubated at the temperatures indicated.

larly. At $37^{\circ} \mathrm{C}$, the control cells harboring the empty vector failed to grow, whereas the cells expressing either one of the yeast ISWI genes grew (Fig. 6B). This result demonstrated clearly that the temperature-sensitive phenotype requires mutations in all three genes. Consistent with the phenotypes of the null mutants described above, the ISW1 gene rescued the phenotype more efficiently than the ISW2 gene as judged by the colony size. The cells expressing both ISW1 and ISW2 grew slightly better than those expressing only ISW1. In a striking contrast, the ISW1-K227A or ISW2-K214A genes were totally inactive in rescuing the synthetic temperature-sensitive phenotype of the triple mutant. Yeast cells harboring the mutant genes were indistinguishable from those harboring an empty vector. This result indicates that the ATP-dependent activities of the yeast ISWI complexes, such as nucleosome disruption and/or spacing activities, are essential for their in vivo functions. Similarly, these mutants also failed to rescue formamide-sensitive phenotypes of the isw1 isw2 chd1 strain (data not shown).

\section{Discussion}

In this study we identified two ISWI genes, ISW1 and 
ISW2, in the yeast $S$. cerevisiae. ISW1p forms a foursubunit complex, which possesses nucleosome-stimulated ATPase activity, nucleosome disruption activity, and nucleosome spacing activity. ISW2p forms a twosubunit complex that does not share any common subunits with ISW1 complex. ISW2 complex has a nucleosome-stimulated ATPase activity and a nucleosome spacing activity, but does not show nucleosome disruption activity in our assays. These two complexes are the first nucleosome spacing factors identified in yeast. When the yeast ISWI genes were mutated together with the CHD1 gene, the yeast cells show synthetic lethality under environmental stresses, demonstrating their strong genetic interactions.

\section{Yeast ISWI proteins form separate complexes} with distinct biochemical activities

Both of the ISWI genes in S. cerevisiae encode subunits of ATP-dependent chromatin remodeling complexes. However, the biochemical properties of the two complexes are distinct. The differences in their biochemical activities must reflect differences in their mechanisms of action. ISW1 and ISW2 complexes created distinct nucleosome spacing in all conditions tested. The mechanisms by which yeast ISWI complexes facilitate regular spacing of nucleosomes remain to be determined. One possible model is that ISWI complexes weaken histoneDNA contacts, which facilitates nucleosomes to find thermodynamically stable positions (Ito et al. 1997; Alexiadis et al. 1998). If the arrays of nucleosomes are stacked into higher order structure, this may lead to regular spacing of nucleosomes. If this is the case, ISW1 and ISW2 complexes may form distinct higher order folding of nucleosome arrays, which may result in formation of distinct nucleosome spacing. The stoichiometry of the complexes to nucleosomes in our assays (1-200 nucleosomes to 1 complex) is not consistent with the possibility that the complexes function as structural components of nucleosome arrays. It is tempting to imagine these complexes being recruited to distinct loci on chromosomes that require distinct spacing of nucleosomes in vivo.

Another difference in the two yeast ISWI complexes is that ISW1 complex demonstrated nucleosome disruption activity on the Drosophila hsp70 promoter, whereas ISW2 complex failed to do so under the same conditions. Although ISW2 complex may truly lack nucleosome disruption activity, it is also possible that specific transcription factors and/or promoters are required for its nucleosome disruption activity. To address this possibility, it is essential to identify genes regulated by ISW2 complex in vivo. It will also be more informative to use yeast core histones in biochemical analyses when technical difficulties in obtaining a large quantity of yeast core histones are solved in the future. However, the nucleosome disruption assays clearly demonstrated that ISW1 and ISW2 complexes have distinct biochemical activities.

It is interesting that there are two copies each of genes that belong to the SWI2/SNF2 and ISWI subfamilies (Eisen et al. 1995) in both humans and yeast (human SWI2/ SNF2, hbrm and BGR-1; human ISWI, hSNF2L and hSNF2h; yeast SWI2/SNF2, SWI2/SNF2 and STH1; yeast ISWI, ISW1 and ISW2). In mammalian systems, biochemical activities of BRG1 and hbrm complexes are indistinguishable (Kwon et al. 1994; Wang et al. 1996a). However, BRG1 and hbrm are differentially regulated on mitogen-induction of cell growth and ras-mediated oncogenic transformation of cells (Muchardt et al. 1998), demonstrating that they may have distinct sets of target genes. Yeast complexes containing SWI2/SNF2p and STH1p have similar biochemical activities (Kwon et al. 1994; Cairns et al. 1996) but have clearly distinct functions in vivo, as SWI2/SNF2 is nonessential (Neigeborn and Carlson 1984; Stern et al. 1984) whereas STH1 is essential (Laurent et al. 1992; Tsuchiya et al. 1992; Cairns et al. 1996). Our present study demonstrated that ISW1 and ISW2 complexes have distinct biochemical activities, suggesting that they may have distinct functions in vivo. These data indicate that multiple ATP-dependent chromatin remodeling complexes with distinct functions are required in both lower and higher eukaryotes.

\section{Yeast ISWI genes show genetic interactions}

In this paper we report the first observation of genetic interactions among genes encoding the subunits of ATPdependent chromatin-remodeling factors. Our genetic experiments revealed that at least one of the three ISWIrelated genes (ISW1, ISW2, or CHD1) is necessary for yeast cells to survive stress conditions. This data implies that in vivo at least one of the ATP-dependent chromatin-remodeling complexes is necessary to establish and/ or maintain proper chromatin structure that is essential for cells to survive demanding conditions. It is of particular interest that ISW1 and ISW2 show genetic interactions with CHD1. The CHD1 gene is highly conserved from yeast to humans (Stokes and Perry 1995; Stokes et al. 1996; Woodage et al. 1997). In flies, polytene chromosome staining using anti-CHD1 antibody has demonstrated a striking localization of the protein within the interbands (Stokes et al. 1996). The staining was also seen on some, but not all, puffs. These data suggest that CHD1 has a positive role in transcription or in the establishment of active chromatin structure. In yeast, the chd1 mutant shows a 6-azauracil-resistant phenotype, which may suggest that CHD1 is involved in the regulation of transcription (Woodage et al. 1997). These reports and the genetic interactions of the ISW1, ISW2, and CHD1 genes in this study are consistent with a possibility that ISW1 and ISW2 complexes play roles in the regulation of transcription. Our genetic data prompt us to characterize the biochemical properties of CHD1 complex in the near future to test if it also has the ability to alter chromatin structure. Interestingly, we have not observed any genetic interactions among ISW1, ISW2, and SWI2/SNF2 genes so far (T. Tsukiyama, unpubl.), which 
may suggest distinct functions of SWI2/SNF2 and ISWI genes in yeast.

The mechanism underlying the synthetic stress-sensitive phenotypes of the isw1 isw2 chd1 triple mutant remains as an intriguing question. ISW1, ISW2 and CHD1 complexes may redundantly regulate overlapping sets of target genes that enable cells to survive stress conditions. It is also possible that they regulate distinct sets of genes. In this case, these genes may be involved in stress resistance in either redundant or additive fashion. In any case, the fact that the ATP-dependent activities of the yeast ISWI complexes are necessary to rescue the stresssensitive phenotypes of the mutants strongly suggests that the chromatin-remodeling activity is essential for the in vivo functions of the complexes.

\section{Materials and methods}

\section{Media and strains}

Growth and manipulation of yeast strains was done according to standard procedures (Adams et al. 1997). Yeast strains used in this study are listed in Table 1. All strains are congenic to W303 (Thomas and Rothstein 1989) except that a weak rad5 mutation in the original W303-1A and W303-1B strains was repaired (Zhao et al. 1998).

\section{Plasmids}

Plasmid pEMBL-ISW1 was constructed by inserting the ORF of the ISW1 gene under the GAL1-CYC1 promoter of pEMBLyex4

Table 1. Yeast strains used in this study

\begin{tabular}{ll}
\hline Strain & \multicolumn{1}{c}{ Genotype } \\
\hline W303-1A & $\begin{array}{c}\text { MATa } \text { ade2-1 his3-11,15 leu2-3,112 trp1-1 ura3-1 } \\
\text { can1-100 }\end{array}$ \\
W1588-4C & same as W303-1A but RAD5 \\
W1588-4A & same as W1588-4C but MATd \\
JPY17 & same as W1588-4A but chd1::TRP1 \\
YTT128 & same as W303-1A but isw1::ADE2 \\
& [pEMBL-ISW1] \\
YTT186 & same as W1588-4C but isw1::ADE2 \\
YTT196 & same as W1588-4A but isw2::LEU2 \\
YTT199 & same as W1588-4C but isw1::ADE2 isw2::LEU2 \\
YTT223 & same as W1588-4C but isw1:ADE2 chd1::TRP1 \\
YTT225 & same as W1588-4C but isw2::LEU2 chd1::TRP1 \\
YTT227 & same as W1588-4C but isw1:ADE2 isw2::LEU2 \\
& chd1::TRP1 \\
YTT395 & same as YTT196 but pep4::HIS3 [pRS416-ISW2] \\
YTT416 & same as YTT186 but pep4::HIS3 [pRS416-ISW1] \\
YTT417 & same as YTT186 but pep4::HIS3 \\
& [pRS416-ISW1-K227A] \\
YTT418 & same as YTT196 pep4::HIS3 \\
& [pRS416-ISW2-K214A] \\
YTT419 & same as W1588-4C but [pRS416] \\
YTT420 & same as YTT227 but [pRS416] \\
YTT421 & same as YTT227 but [pRS416-ISW1] \\
YTT422 & same as YTT227 but [pRS416-ISW2] \\
YTT423 & same as YTT227 but [pRS416-ISW1, ISW2] \\
YTT424 & same as YTT227 but [pRS416-ISW1-K227A] \\
YTT425 & same as YTT227 but [pRS416-ISW2-K214A] \\
\hline
\end{tabular}

vector (Cesarei and Murray 1987). The ISW1 gene in this construct was tagged with the Flag epitope (Asp-Tyr-Lys-Asp-AspAsp-Asp-Lys) and a $6 \times$ histidine at the $5^{\prime}$ and $3^{\prime}$ terminals of the gene, respectively. To construct pRS416-ISW1, two copies of the Flag sequence followed by a termination codon and a PstI restriction site were inserted at the $3^{\prime}$ end of the ORF of ISW1 by PCR. A BamHI (-853)-PstI fragment was cloned into pRS416. Similarly, two copies of the Flag sequence were inserted at the 3' end of the ISW2 gene and an EcoRI (-948)-PstI fragment was cloned into pRS416 to create pRS416-ISW2. Oligonucleotide-mediated site-directed mutagenesis was used to introduce K227A and K214A mutations within the ATP-binding sites of the ISW1 and ISW2 genes to create pRS416-ISW1K227A and pRS416-ISW2-K214A plasmids. After epitope tagging and mutagenesis, the entire DNA fragments used for manipulations were sequenced to eliminate possible introduction of secondary mutations during manipulations.

\section{Purification of the ISWI complexes}

NURF was purified from 0- to 12-hr Drosophila embryos through P11 fractions as previously described (Tsukiyama and Wu 1995).

The wild-type ISW1 complex was purified from YTT128 and YTT416, which produced indistinguishable complexes. ISW1K227A complex was purified from YTT424. The wild-type ISW2 complex and ISW2-K214A complex were purified form YTT422 and YTT425, respectively. Yeast cells were grown in a synthetic medium lacking uracil until $\mathrm{OD}_{600}=\sim 5$ in a 40-liter fermentor (New Brunswick). Yeast cells were harvested, washed once in ice-cold water, twice in ice-cold buffer H-0.3 (buffer H: $25 \mathrm{~mm}$ HEPES-KOH at $\mathrm{pH} 7.6,0.5 \mathrm{~mm}$ EGTA, $0.1 \mathrm{~mm}$ EDTA, $2 \mathrm{~mm}$ $\mathrm{MgCl}_{2}, 20 \%$ glycerol, $0.02 \% \mathrm{NP}-40,1 \mathrm{~mm}$ DTT, $0.5 \mathrm{~mm} \mathrm{Na}$ metabisulfite, $1 \mathrm{mM}$ PMSF, $2 \mu \mathrm{M}$ pepstatin A, $0.6 \mu \mathrm{M}$ leupeptin, $2 \mathrm{~mm}$ benzamidine, $2 \mu \mathrm{g} / \mathrm{ml}$ chimostatin; the number following buffer $\mathrm{H}$ denotes the molar concentration of $\mathrm{KCl}$ ), and frozen in liquid nitrogen as 'noodles' as described previously (Schultz et al. 1997). Whole cell extract was prepared either by mortar and pestle (Brinkman) (Schultz et al. 1997) or by microfluidizer (Microfluidics Corp.) in buffer H-0.5. The extracts were spun at $37,000 \mathrm{rpm}$ for $90 \mathrm{~min}$ in an SW40 rotor (Beckman) to remove insoluble materials.

For some preparations, whole cell extracts from 30-100 grams of cells were fractionated over a 40-ml Bio-Rex 70 column. For the ISW1 complex, the salt concentration of the extract was adjusted to $0.2 \mathrm{M} \mathrm{KCl}$ and loaded and washed by buffer H-0.2. The column was then eluted with a linear gradient from 0.2 to $0.6 \mathrm{M} \mathrm{KCl}$ in buffer $\mathrm{H}$. For the ISW2 complex, the extract was adjusted to $0.15 \mathrm{M} \mathrm{KCl}$ and loaded and washed by buffer H-0.15. The column was eluted with a linear gradient from 0.15 to 0.6 $\mathrm{M} \mathrm{KCl} \mathrm{in} \mathrm{buffer} \mathrm{H}$. The fractions containing the yeast ISWI proteins were identified by Western blotting using Flag M2 antibody (Kodak IBI). Flag M2 beads $(400 \mu \mathrm{l})$ were added to pooled fractions from the Bio-Rex column and incubated at $4^{\circ} \mathrm{C}$ for $3 \mathrm{hr}$ with gentle mixing. The beads were washed twice with $15 \mathrm{ml}$ of buffer $\mathrm{H}-0.5$, six times with $1 \mathrm{ml}$ of buffer $\mathrm{H}-0.5$, three times with $1 \mathrm{ml}$ of buffer H-0.1, and eluted four times with $2 \mathrm{mg} / \mathrm{ml}$ Flag peptide in $400 \mu \mathrm{l}$ of buffer H-0.1 for $30 \mathrm{~min}$ each. For both the ISW1 and ISW2 complexes, the fractions from Flag beads were then loaded onto either $1 \mathrm{ml}$ of Mono Q or $0.2 \mathrm{ml}$ of Source Q column (Pharmacia) in buffer H-0.1. The column was washed with buffer H-0.1 and eluted by a linear gradient from 0.1 to 0.6 $\mathrm{M} \mathrm{KCl}$ in buffer $\mathrm{H}$. We later found that the Flag bead purification can be done before the Bio-Rex column. In this case, purification by Flag beads was done as described above, and the bed volume 
of the Bio-Rex column was reduced to $0.2 \mathrm{ml}$. The ISW1-GAT complex was purified by Bio-Rex column followed by Flag beads. All chromatographic manipulations except for the Flag columns were done using Pharmacia FPLC system. To quantify the yeast ISWI complexes, the concentration of yeast ISWI proteins in the most purified fractions was estimated by SDS/ PAGE followed by silver staining using BSA and purified recBCD enzyme (Taylor and Smith 1995) as controls.

\section{ATPase assays}

ATPase assays of purified yeast ISWI complexes were done as described (Tsukiyama and Wu 1995) except that 15 fmoles (Fig. 2) or 7.5 fmoles (Fig. 5B) of the yeast ISWI complexes, $0.1 \mathrm{~mm}$ cold ATP, $5 \mathrm{mM} \mathrm{MgCl}_{2}$, was used in a 5- $\mu$ l reaction volume. The reaction mixture was incubated for 30 (Fig. 2) or 60 (Fig. 5B) min at $30^{\circ} \mathrm{C}$, and $0.5 \mu \mathrm{l}$ was spotted onto thin layer chromatography plates. ATP and free phosphate were separated in $0.8 \mathrm{M} \mathrm{LiCl}, 0.8$ $M$ acetic acid, localized by autoradiograph, and quantified by scintillation counting.

\section{Chromatin assembly and disruption assays}

Chromatin assembly on plasmid pdhspXX3.2, Sarkosyl treatment, and purification of chromatin through gel-filtration column was done as described previously (Tsukiyama and Wu 1995), except that Sepharose CL-4B was used instead of BioGel A1.5m (Mizuguchi et al. 1997). Disruption assay for nucleosome arrays by MNase digestion and chromatin accessibility assay by restriction enzyme digestion were carried out as described (Tsukiyama and Wu 1995) using 20 and 10 fmoles of the yeast ISW1 and ISW2 complexes, respectively, at $30^{\circ} \mathrm{C}$.

\section{Nucleosome spacing assays}

Standard nucleosome spacing assay reactions contained $3 \mathrm{~mm}$ ATP and its regeneration system (McNAP) (Becker et al. 1994), purified recombinant yeast NAP-1 $(0.75 \mu \mathrm{g})$ (Fujii-Nakata et al. 1992), purified core histones from Drosophila embryos (0.25 $\mu \mathrm{g})$, the yeast ISWI complexes ( 15 fmoles), and $\lambda$ phage DNA $(0.25$ $\mu \mathrm{g}$ ) in $10 \mathrm{~mm}$ HEPES-KOH (pH 7.6), $50 \mathrm{~mm} \mathrm{KCl}, 5 \mathrm{~mm} \mathrm{MgCl}_{2}$, $0.5 \mathrm{~mm}$ EGTA, $10 \%$ glycerol, and $0.1 \mu \mathrm{g} / \mu \mathrm{l} \mathrm{BSA}$. The reaction was incubated at $30^{\circ} \mathrm{C}$ for $4 \mathrm{hr}$, and digested with MNase as described (Becker et al. 1994; Tsukiyama and Wu 1996). DNA was purified and separated through $1.3 \%$ agarose gel.

\section{Acknowledgments}

We are grateful to M. Gelbart, S. Hahn, D. Gottschling, and J. Tamkun for critical reading of the manuscript. We thank D. Gottchling, M. Lichten, A. Goldman, S. Bohen, and F. Winston for technical advice and continuous encouragement. We also thank M. Steinberg for making subclones of ISW1 gene for sitedirected mutagenesis, M. Schults for protocol for yeast extract preparation before publication, R. Rothstein for yeast strains, and A. Taylor for purified Rec BCD complex. T.T. is especially grateful to all lecturers and teaching assistants at the 1997 Cold Spring Harbor Yeast Genetics Course. This work was supported in part by grant 91-020-06-IRG from the American Cancer Society and a Pew Charitable Trust Biomedical Scholars Fellowship to T.T.

The publication costs of this article were defrayed in part by payment of page charges. This article must therefore be hereby marked 'advertisement' in accordance with 18 USC section 1734 solely to indicate this fact.

\section{References}

Aasland, R., A.F. Stewart, and T. Gibson. 1996. The SANT domain: A putative DNA-binding domain in the SWI-SNF and ADA complexes, the transcriptional co-repressor N-CoR and TFIIIB. Trends Biochem. Sci. 21: 87-88.

Adams, A., D. Gottschling, and T. Stearns. 1997. Methods in yeast genetics. Cold Spring Harbor Laboratory Press, Cold Spring Harbor, NY.

Alexiadis, V., P.D. Varga-Weisz, E. Bonte, P.B. Becker, and C. Gruss. 1998. In vitro chromatin remodelling by chromatin accessibility complex (CHRAC) at the SV40 origin of DNA replication. $E M B O J$. 17: 3428-3438.

Armstrong, J.A. and B.M. Emerson. 1998. Transcription of chromatin: These are complex times. Curr. Opin. Genet. Dev. 8: $165-172$.

Becker, P.B. and C. Wu. 1992. Cell-free system for assembly of transcriptionally repressed chromatin from Drosophila embryos. Mol. Cell. Biol. 12: 2241-2249.

Becker, P.B., T. Tsukiyama, and C. Wu. 1994. Chromatin assembly extracts from Drosophila embryos. Methods Cell Biol. 44: 207-223.

Cairns, B.R. 1998. Chromatin remodeling machines: Similar motors, ulterior motives. Trends Biochem. Sci. 23: 20-25.

Cairns, B.R., Y. Lorch, Y. Li, M. Zhang, L. Lacomis, H. Erdjument-Bromage, P. Tempst, J. Du, B. Laurent, and R.D. Kornberg. 1996. RSC, an essential, abundant chromatin-remodeling complex. Cell 87: 1249-1260.

Cesarei, G. and J.A.H. Murray. 1987. Plasmid vectors carrying the replication origin of filamentous single-stranded phages. In Genetic engineering: Principles and methods (ed. J.K. Setlow and A. Hollaender), pp. 135-154. Plenum Press, New York, NY.

Cote, J., J. Quinn, J.L. Workman, and C.L. Peterson. 1994. Stimulation of GAL4 derivative binding to nucleosomal DNA by the yeast SWI/SNF complex. Science 265: 53-60.

Eisen, J.A., K.S. Sweder, and P.C. Hanawalt. 1995. Evolution of the SNF2 family of proteins: Subfamilies with distinct sequences and functions. Nucleic Acids Res. 23: 2715-2723.

Elfring, L.K., R. Deuring, C.M. McCallum, C.L. Peterson, and J.W. Tamkun. 1994. Identification and characterization of Drosophila relatives of the yeast transcriptional activator SNF2/SWI2. Mol. Cell. Biol. 14: 2225-2234.

Fan, Q.Q. and T.D. Petes. 1996. Relationship between nucleasehypersensitive sites and meiotic recombination hot spot activity at the HIS4 locus of Saccharomyces cerevisiae. Mol. Cell. Biol. 16: 2037-2043.

Felsenfeld, G. 1996. Chromatin unfolds. Cell 86: 13-19.

Fujii-Nakata, T., Y. Ishimi, A. Okuda, and A. Kikuchi. 1992. Functional analysis of nucleosome assembly protein, NAP1. The negatively charged $\mathrm{COOH}$-terminal region is not necessary for the intrinsic assembly activity. J. Biol. Chem. 267: 20980-20986.

Gaillard, P.H., E.M. Martini, P.D. Kaufman, B. Stillman, E. Moustacchi, and G. Almouzni. 1996. Chromatin assembly coupled to DNA repair: A new role for chromatin assembly factor I. Cell 86: 887-896.

Gottschling, D.E., O.M. Aparicio, B.L. Billington, and V.A. Zakian. 1990. Position effect at S. cerevisiae telomeres: Reversible repression of Pol II transcription. Cell 63: 751-762.

Imbalzano, A.N., H. Kwon, M.R. Green, and R.E. Kingston. 1994. Facilitated binding of TATA-binding protein to 
nucleosomal DNA. Nature 370: 481-485.

Ito, T., M. Bulger, R. Kobayashi, and J.T. Kadonaga. 1996. Drosophila NAP-1 is a core histone chaperone that functions in ATP-facilitated assembly of regularly spaced nucleosomal arrays. Mol. Cell. Biol. 16: 3112-3124.

Ito, T., M. Bulger, M.J. Pazin, R. Kobayashi, and J.T. Kadonaga. 1997. ACF, an ISWI-containing and ATP-utilizing chromatin assembly and remodeling factor. Cell 90: 145-155.

Kadonaga, J.T. 1998. Eukaryotic transcription: An interlaced network of transcription factors and chromatin-modifying machines. Cell 92: 307-313.

Kaufman, P.D., R. Kobayashi, and B. Stillman. 1997. Ultraviolet radiation sensitivity and reduction of telomeric silencing in Saccharomyces cerevisiae cells lacking chromatin assembly factor-I. Genes \& Dev. 11: 345-357.

Khavari, P.A., C.L. Peterson, J.W. Tamkun, D.B. Mendel, and G.R. Crabtree. 1993. BRG1 contains a conserved domain of the SWI2/SNF2 family necessary for normal mitotic growth and transcription. Nature 366: 170-174.

Kingston, R.E., C.A. Bunker, and A.N. Imbalzano. 1996. Repression and activation by multiprotein complexes that alter chromatin structure. Genes \& Dev. 10: 905-920.

Kornberg, R.D. and Y. Lorch. 1995. Interplay between chromatin structure and transcription. Curr. Opin. Cell. Biol. 7: 371-375

Krude, T. and S.C.R. Elgin. 1996. Pushing nucleosomes around. Chromatin. Curr. Biol. 6: 511-515.

Kwon, H., A.N. Imbalzano, P.A. Khavari, R.E. Kingston, and M.R. Green. 1994. Nucleosome disruption and enhancement of activator binding by a human SW1/SNF complex. Nature 370: 477-481.

Laurent, B.C., X. Yang, and M. Carlson. 1992. An essential Saccharomyces cerevisiae gene homologous to SNF2 encodes a helicase-related protein in a new family. Mol. Cell. Biol. 12: $1893-1902$.

Laurent, B.C., I. Treich, and M. Carlson. 1993. The yeast SNF2/ SWI2 protein has DNA-stimulated ATPase activity required for transcriptional activation. Genes \& Dev. 7: 583-591.

LeRoy, G., G. Orphanides, W.S. Lane, and D. Reinberg. 1998. Requirement of RSF and FACT for transcription of chromatin templates in vitro. Science 282: 1900-1904.

Luger, K., A.W. Mader, R.K. Richmond, D.F. Sargent, and T.J. Richmond. 1997. Crystal structure of the nucleosome core particle at 2.8 A resolution. Nature 389: 251-260.

Mizuguchi, G., T. Tsukiyama, J. Wisniewski, and C. Wu. 1997. Role of nucleosome remodeling factor NURF in transcriptional activation of chromatin. Mol. Cell 1: 141-150.

Mizuno, K., Y. Emura, M. Baur, J. Kohli, K. Ohta, and T. Shibata. 1997. The meiotic recombination hot spot created by the single-base substitution ade6-M26 results in remodeling of chromatin structure in fission yeast. Genes \& Dev. 11: 876886.

Muchardt, C., B. Bourachot, J.C. Reyes, and M. Yaniv. 1998. ras transformation is associated with decreased expression of the brm/SNF2 $\alpha$ ATPase from the mammalian SWI-SNF complex. EMBO I. 17: 223-231.

Neigeborn, L. and M. Carlson. 1984. Genes affecting the regulation of SUC2 gene expression by glucose repression in Saccharomyces cerevisiae. Genetics 108: 845-858.

Pazin, M.J. and J.T. Kadonaga. 1997. SWI2/SNF2 and related proteins: ATP-driven motors that disrupt protein-DNA interactions? Cell 88: 737-740.

Peterson, C.L. 1996. Multiple SWItches to turn on chromatin? Curr. Opin. Genet. Dev. 6: 171-175.

Rothstein, R. 1991. Targeting, disruption, replacement, and allele rescue: Integrative DNA transformation in yeast. Meth- ods Enzymol. 194: 281-301.

Schultz, M.C., D.J. Hockman, T.A. Harkness, W.I. Garinther, and B.A. Altheim. 1997. Chromatin assembly in a yeast whole-cell extract. Proc. Natl. Acad. Sci. 94: 9034-9039.

Stanhope-Baker, P., K.M. Hudson, A.L. Shaffer, A. Constantinescu, and M.S. Schlissel. 1996. Cell type-specific chromatin structure determines the targeting of $\mathrm{V}(\mathrm{D}) \mathrm{J}$ recombinase activity in vitro. Cell 85: 887-897.

Steger, D.J. and J.L. Workman. 1996. Remodeling chromatin structures for transcription: What happens to the histones? BioEssays 18: 875-884.

Stern, M., R. Jensen, and I. Herskowitz. 1984. Five SWI genes are required for expression of the $\mathrm{HO}$ gene in yeast. J. Mol. Biol. 178: $853-868$.

Stokes, D.G. and R.P. Perry. 1995. DNA-binding and chromatin localization properties of CHD1. Mol. Cell. Biol. 15: 27452753.

Stokes, D.G., K.D. Tartof, and R.P. Perry. 1996. CHD1 is concentrated in interbands and puffed regions of Drosophila polytene chromosomes. Proc. Natl. Acad. Sci. 93: 7137-7142.

Svaren, J. and W. Horz. 1996. Regulation of gene expression by nucleosomes. Curr. Opin. Genet. Dev. 6: 164-170.

Tamkun, J.W., R. Deuring, M.P. Scott, M. Kissinger, A.M. Pattatucci, T.C. Kaufman, and J.A. Kennison. 1992. brahma: A regulator of Drosophila homeotic genes structurally related to the yeast transcriptional activator SNF2/SWI2. Cell 68: $561-572$.

Taylor, A.F. and G.R. Smith. 1995. Monomeric RecBCD enzyme binds and unwinds DNA. J. Biol. Chem. 270: 2445124458.

Thomas, B.J. and R. Rothstein. 1989. The genetic control of direct-repeat recombination in Saccharomyces: The effect of rad52 and rad1 on mitotic recombination at GAL10, a transcriptionally regulated gene. Genetics 123: 725-738.

Tsuchiya, E., M. Uno, A. Kiguchi, K. Masuoka, Y. Kanemori, S. Okabe, and T. Mikayawa. 1992. The Saccharomyces cerevisiae NPS1 gene, a novel CDC gene which encodes a $160 \mathrm{kDa}$ nuclear protein involved in G2 phase control. EMBO $\mathrm{J}$. 11: 4017-4026.

Tsukiyama, T. and C. Wu. 1995. Purification and properties of an ATP-dependent nucleosome remodeling factor. Cell 83: 1011-1020.

1996. Purification of GAGA factor of Drosophila and its role in nucleosome disruption. Methods Enzymol. 274: 291299.

1997. Chromatin remodeling and transcription. Curr. Opin. Genet. Dev. 7: 182-191.

Tsukiyama, T., P.B. Becker, and C. Wu. 1994. ATP-dependent nucleosome disruption at a heat-shock promoter mediated by binding of GAGA transcription factor. Nature 367: 525532.

Tsukiyama, T., C. Daniel, J. Tamkun, and C. Wu. 1995. ISWI, a member of the SWI2/SNF2 ATPase family, encodes the 140 $\mathrm{kDa}$ subunit of the nucleosome remodeling factor. Cell 83: 1021-1026.

Tsukamoto, Y., J. Kato, and H. Ikeda. 1997. Silencing factors participate in DNA repair and recombination in Saccharomyces cerevisiae. Nature 388: 900-903.

van Holde, K.E. 1988. Chromatin (ed. A. Rich), pp. 16-30. Springer-Verlag, New York, NY.

Varga-Weisz, P.D., M. Wilm, E. Bonte, K. Dumas, M. Mann, and P.B. Becker. 1997. Chromatin-remodelling factor CHRAC contains the ATPases ISWI and topoisomerase II. Nature 388: 598-602.

Wade, P.A., P.L. Jones, D. Vermaak, and A.P. Wolffe. 1998. A multiple subunit Mi-2 histone deacetylase from Xenopus 
laevis cofractionates with an associated Snf2 superfamily ATPase. Curr. Biol. 8: 843-846.

Wang, W., J. Cote, Y. Xue, S. Zhou, P.A. Khavari, S.R. Biggar, C. Muchardt, G.V. Kalpana, S.P. Goff, M. Yaniv, J.L. Workman, and G.R. Crabtree. 1996a. Purification and biochemical heterogeneity of the mammalian SWI-SNF complex. EMBO $\mathrm{T}$. 15: 5370-5382.

Wang, W., Y. Xue, S. Zhou, A. Kuo, B.R. Cairns, and G.R. Crabtree. 1996b. Diversity and specialization of mammalian SWI/SNF complexes. Genes \& Dev. 10: 2117-2130.

Wolffe, A.P., J. Wong, Q. Li, B.Z. Levi, and Y.B. Shi. 1997. Three steps in the regulation of transcription by the thyroid hormone receptor: Establishment of a repressive chromatin structure, disruption of chromatin and transcriptional activation. Biochem. Soc. Trans. 25: 612-615.

Woodage, T., M.A. Basrai, A.D. Baxevanis, P. Hieter, and F.S. Collins. 1997. Characterization of the CHD family of proteins. Proc. Natl. Acad. Sci. 94: 11472-11477.

Wu, T.C. and M. Lichten. 1994. Meiosis-induced double-strand break sites determined by yeast chromatin structure. Science 263: 515-518.

Zhang, Y., G. LeRoy, H.P. Seelig, W.S. Lane, and D. Reinberg. 1998. The dermatomyositis-specific autoantigen Mi2 is a component of a complex containing histone deacetylase and nucleosome remodeling activities. Cell 95: 279-289.

Zhao, X., E.G. Muller, and R. Rothstein. 1998. A suppressor of two essential checkpoint genes identifies a novel protein that negatively affects dNTP pools. Mol. Cell 2: 329-340. 


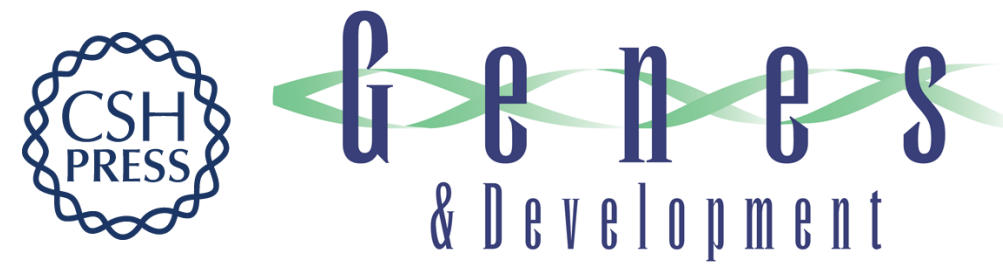

\section{Characterization of the Imitation Switch subfamily of ATP-dependent chromatin-remodeling factors in Saccharomyces cerevisiae}

Toshio Tsukiyama, Jeffrey Palmer, Carolyn C. Landel, et al.

Genes Dev. 1999, 13:

References This article cites 62 articles, 24 of which can be accessed free at:

http://genesdev.cshlp.org/content/13/6/686.full.html\#ref-list-1

License

Email Alerting

Receive free email alerts when new articles cite this article - sign up in the box at the top

Service

right corner of the article or click here.

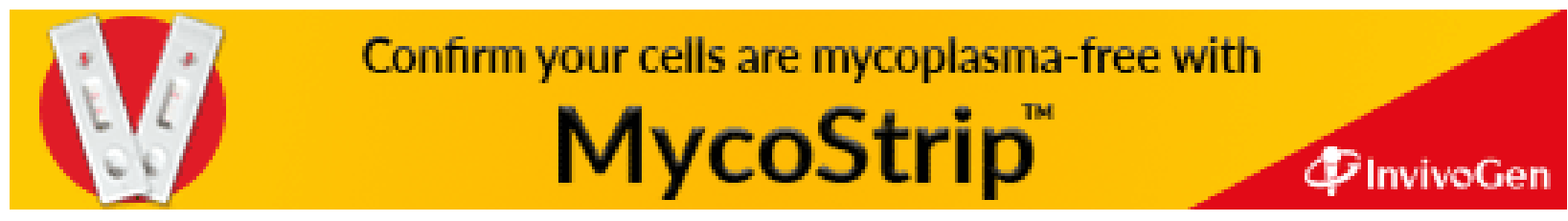

